\title{
Modeling the e-APD SAPHIRA/C-RED ONE camera at low flux level
}

\section{An attempt to count photons in the near-infrared with the MIRC-X interferometric combiner}

\author{
C. Lanthermann ${ }^{1}$, N. Anugu ${ }^{2}$, J.-B. Le Bouquin ${ }^{1,3}$, J. D. Monnier ${ }^{3}$, S. Kraus², and K. Perraut ${ }^{1}$ \\ ${ }^{1}$ Univ. Grenoble Alpes, CNRS, IPAG, 38000 Grenoble, France \\ e-mail: cyprien.lanthermann@univ-grenoble-alpes.fr \\ 2 University of Exeter, School of Physics and Astronomy, Stocker Road, Exeter, EX4 4QL, UK \\ 3 University of Michigan, Department of Astronomy, 1085 S. University Ave, West Hall 323, Ann Arbor, MI 48109, USA
}

Received 10 January 2019 / Accepted 15 March 2019

\begin{abstract}
Context. We implement an electron avalanche photodiode (e-APD) in the MIRC-X instrument, which is an upgrade of the sixtelescope near-infrared imager MIRC, at the CHARA array. This technology should improve the sensitivity of near-infrared interferometry.

Aims. We aim to characterize a near-infrared C-RED ONE camera from First Light Imaging (FLI) using an e-APD from Leonardo (previously SELEX).

Methods. We first used the classical mean-variance analysis to measure the system gain and the amplification gain. We then developed a physical model of the statistical distribution of the camera output signal. This model is based on multiple convolutions of the Poisson statistic, the intrinsic avalanche gain distribution, and the observed distribution of the background signal. At low flux level, this model independently constrains the incident illumination level, the total gain, and the excess noise factor of the amplification. Results. We measure a total transmission of $48 \pm 3 \%$ including the cold filter and the Quantum Efficiency. We measure a system gain of $0.49 \mathrm{ADU} / \mathrm{e}$, a readout noise of $10 \mathrm{ADU}$, and amplification gains as high as 200. These results are consistent between the two methods and therefore validate our modeling approach. The measured excess noise factor based on the modeling is $1.47 \pm 0.03$, with no obvious dependency with flux level or amplification gain.

Conclusions. The presented model allows the characteristics of the e-APD array to be measured at low flux level independently of a preexisting calibration. With $<0.3$ electron equivalent readout noise at kilohertz frame rates, we confirm the revolutionary performances of the camera with respect to the PICNIC or HAWAII technologies. However, the measured excess noise factor is significantly higher than what is claimed in the literature $(<1.25)$, and explains why counting multiple photons remains challenging with this camera.
\end{abstract}

Key words. instrumentation: detectors - techniques: high angular resolution - methods: data analysis - infrared: general

\section{Introduction}

Optical long baseline interferometry allows for imaging at higher angular resolution than classical monolithic telescopes for a given wavelength. For instance, the gain in resolution is about a factor 3 (resp., 8) between a diffraction-limited $40 \mathrm{~m}$ singledish telescope and the Very Large Telescope Interferometer (VLTI, Haguenauer et al. 2012) (resp., the CHARA array; Gies et al. 2018). As it is a natural trade-off between the damaging effect of atmospheric turbulence at shorter wavelengths and the increased sky brightness at longer wavelengths, many interferometric instruments operate in the near-infrared (NIR), like PIONIER and GRAVITY at the VLTI (Le Bouquin et al. 2011; GRAVITY Collaboration 2017): CLIMB, CLASSIC, JouFLU, and MIRC-X at CHARA (Monnier et al. 2006a; ten Brummelaar et al. 2012; Scott et al. 2014). As a typical example, single-mode optical interferometry in the $H$-band $(1.6 \mu \mathrm{m})$ requires individual exposure times of about $10 \mathrm{~ms}$. In this spectral range, to cope with atmospheric effects and to stabilize the interferometric fringe patterns implies measurement and servo-control of the fringe positions during a fraction of the coherence time, that is, during less than a few milliseconds. Fringe tracking in the NIR thus needs fast detectors working at kilohertz speed and allows for the instrument sensitivity to be increased by allowing minutelong exposures to be done on the scientific detector (GRAVITY Collaboration 2017).

In addition, in the $H$-band, the total instrument and sky background is about ten photons per exposure per telescope. Considering that at least $100 \times 10$ pixels are commonly needed to encode the spectrally dispersed fringe signal, the background noise is much lower than one photon per pixel and per exposure. Typical readout noise of PICNIC or HAWAII detectors for such a frame rate and format are approximately eight electrons per exposure and per pixel (Pedretti et al. 2004; Beckmann et al. 2014). Consequently, the detector readout noise has remained the limiting factor for decades.

The situation changed in the 2000s with the development of matrices of electron Avalanche Photo-Diodes (e-APD) in the NIR. These detectors promised to deliver sub-electron readout noise thanks to amplification of the signal before the readout. On the one hand, a French collaboration of industrial (SOFRADIR, CEA-LETI) and academic (IPAG, LAM, ONERA) partners designed and built several prototypes of the so-called RAPID detector (Feautrier et al. 2014; Guieu et al. 2014). On the another hand, the Leonardo company (former SELEX) in collaboration 
with the ESO developed the SAPHIRA detectors to use them in the wavefront sensors and the fringe tracker of the GRAVITY instrument (Finger et al. 2016; GRAVITY Collaboration 2017). This detector was made available as a commercial product, called C-RED ONE, by the First Light Imaging company (Greffe et al. 2016).

Within the framework of the installation of adaptive optics on the CHARA array (ten Brummelaar et al. 2016) and with the aim of increasing the sensitivity of high-angular-resolution imaging in the NIR, we purchased the first two C-RED ONE cameras in 2017. The first one was used to upgrade the $H$-band six-telescope combiner MIRC, now called MIRC-X (Monnier et al. 2012; Kraus et al. 2018), at the focus of the CHARA optical interferometer since mid-2017. The second one will be used in the $K$-band copy of this instrument, called MYSTIC, and is expected to be installed in 2019 at CHARA (Monnier et al. 2018).

The goal of this paper is to measure the characteristics of the e-APD detector of MIRC-X at our typical low flux level and to determine whether or not photon-counting is achievable in realistic operational conditions.

After this introduction, the second section describes the C-RED ONE camera and the e-APD technology. In the third section, we present a classical characterization of the detector noise and amplification of the camera, based on the meanvariance method. In the fourth section, we introduce an innovative model of the signal distribution which allows us to extract the excess noise factor and the gain. The fifth section discusses the obtained results and compares them with the literature, especially the significant discrepancy of the excess noise factor. The paper ends with a brief summary and conclusion.

\section{The C-RED ONE camera}

\section{1. e-APD technology}

The e-APD technology consists in applying a bias voltage in a lower layer of the pixel. The electron generated by the incident photon in the absorption region migrates to the bottom of the pixel. When the electron travels through the multiplication region, it is accelerated by the bias voltage. At some point, the kinetic energy is high enough to ionize an atom of the substrate by a collision. The two electrons are accelerated again, generating new electrons by collision. This avalanche process is explained in more detail in Finger et al. (2010) and Finger et al. (2012).

The avalanche process is a stochastic process. An incident photon can lead to different numbers of final electrons with a given probability distribution. In this paper, we refer to this probability distribution as the avalanche gain distribution $M$. From this amplification gain distribution, we can derive two principal characteristics of the system: the mean avalanche gain and the excess noise factor. The mean avalanche gain is defined by

$G_{\mathrm{av}}=\langle M\rangle$.

The excess noise factor (ENF) is defined by

$\mathrm{ENF}=\frac{\left\langle M^{2}\right\rangle}{\langle M\rangle^{2}}$.

The ENF is the additional noise brought by the avalanche process on the output signal compared to the photon noise obtained without the avalanche process. In the following, we also make use of the mean total gain, defined as

$G=G_{\text {sys }} G_{\text {av }}$,

where $G_{\text {sys }}$ is the system gain in $\mathrm{ADU} / \mathrm{e}$.

\subsection{Camera readout}

Our C-RED ONE cameras (Fig. 1-left) use SAPHIRA MCT SWIR Mark13 e-APD $320 \times 256$ pixels detectors whose overall characteristics are summarized in Table 1.

The camera offers various readout modes. Our custom readout is inspired from the PICNIC camera from the IOTA observatory (Monnier et al. 2006b). First, it consists of multiple consecutive reads of the same pixel (NREADS) before moving to the next pixel on the row. A row is then read multiple times (NLOOPS) before moving to the next row. Those multiple NREADS and NLOOPS are then averaged. It reduces the readout noise and extends the integration time while keeping a somewhat simultaneous integration window for the various pixels of the same row. In the following, we use frame to refer to the process of reading the entire array once, with this combination of NREADS and NLOOPS. Finally, the array is read multiple times nondestructively (NFRAMES_PER_RESET) before the detector is reset and the process starts again. We note that it is possible to read subwindows of the full pixel array. Cropping is possible in the row and column directions and this increases the frame rate.

In most implementations of the on-the-ramp mode, the set of NFRAMES_PER_RESET along the ramp is collapsed into a single value of flux per pixel by the mean of a linear fit. This is not the case in interferometric instruments where a fast frame rate is necessary (Pedretti et al. 2004; Le Bouquin et al. 2011). Instead, we individually analyze the flux of each frame in the ramp by subtracting the measurement of the previous frame.

A typical setup used for on-sky observations consists of reading a window of $320 \times 17$ pixels, with NREADS $=12$ and NLOOPS $=8$. It provides a frame rate of $\approx 355 \mathrm{~Hz}$. The detector is generally reset every 100 frames only. In the experiments of this paper, we used NREADS $=8$ and NLOOPS $=2$ in order to increase the frame rate to $\approx 1916 \mathrm{~Hz}$. This was necessary to lower the background signal per pixel and per frame. Each data set has a total of 2000 frames.

Before being analyzed, we correct the RAW data from a parasitic electronic signal from the pulse tube, which appears as a nearly sinusoidal additive signal; the process is explained in Lanthermann et al. (2018). We then compute the flux per frame, in ADU, by taking the difference between two consecutive frames.

\subsection{Cold optics}

The C-RED ONE camera is delivered in a standalone cryostat, operating at $80 \mathrm{~K}$ thanks to a pulse-tube. It has a cold aperture of $\mathrm{f} / 4$ at about $35 \mathrm{~mm}$ from the detector and $15 \mathrm{~mm}$ from the external surface of the cryostat window (e.g., where the last warm optics can be located). Our MIRC-X camera holds four low-pass cold filters to remove the thermal background; two have a cutoff wavelength at $1.739 \mu \mathrm{m}$, and two at $2.471 \mu \mathrm{m}$. The latter is mandatory to increase the rejection at wavelengths longer than $3 \mu \mathrm{m}$. The theoretical amount of electrons due to the thermal background flux seen by a pixel in a single frame can be computed as follows.

$F_{\mathrm{BKG}_{\text {thermal }}}=\int_{0}^{\infty} B(\lambda, 300 \mathrm{~K}) A \Omega T(\lambda) Q E(\lambda) \frac{1}{f} \mathrm{~d} \lambda$,

where $B(\lambda, 300 \mathrm{~K})$ is the blackbody emission at $300 \mathrm{~K}, A=$ $(24 \mu \mathrm{m})^{2}$ is the surface of a pixel, $\Omega=\pi / 64 \mathrm{str}$ is the solid angle corresponding to the cold aperture, $T(\lambda)$ is the combined transmission of the filters, $Q E(\lambda)$ is the quantum efficiency, and $f=1916 \mathrm{~Hz}$ is the frame rate. We measured the combined transmission and quantum efficiency of the camera 

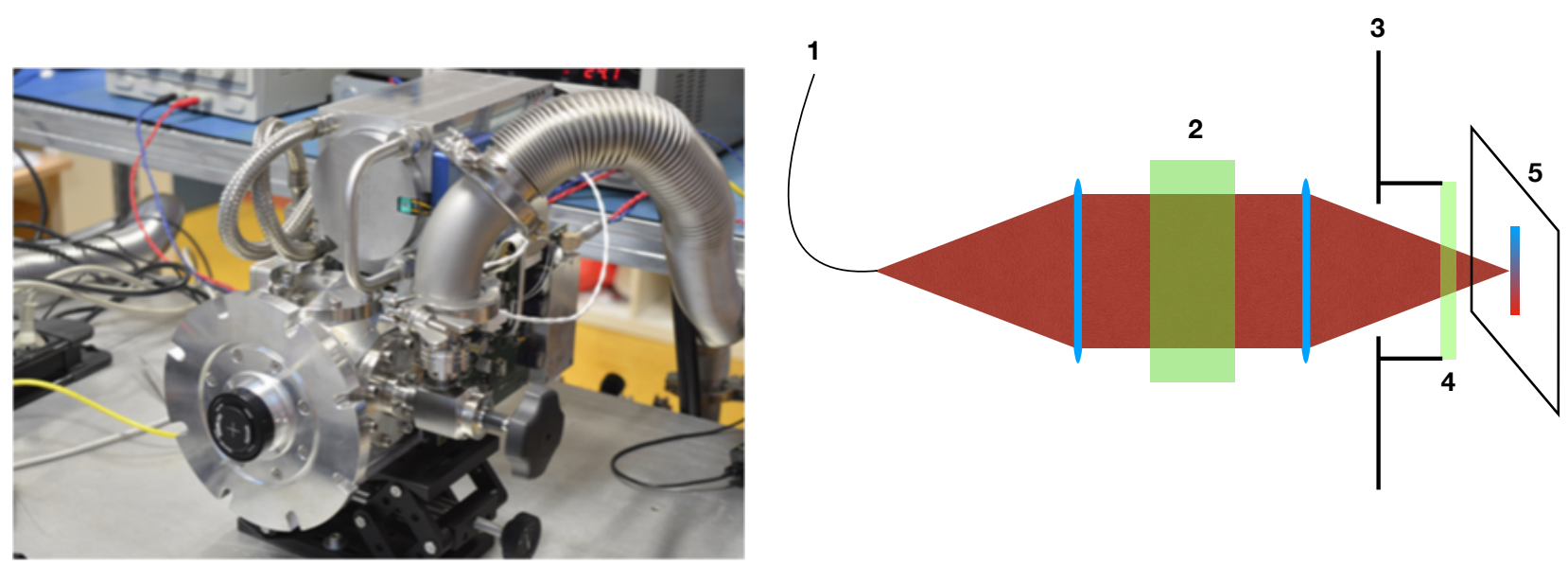

Fig. 1. Left: photograph of the MIRC-X C-RED ONE. Right: scheme of the experimental setup. $1: H$ band single-mode fiber fed by a white-light source; 2: dispersion prism; 3: camera cold stop and baffle; 4: low pass cold filter; 5: detector.

Table 1. Characteristics of the SAPHIRA MCT SWIR Mark13 detector used in our C-RED ONE camera, as specified by the manufacturer.

\begin{tabular}{ll}
\hline \hline Characteristics & Values \\
\hline Format & $320 \times 256$ pixels \\
Detector sensitivity & 0.8 to $3.5 \mu \mathrm{m}$ \\
Readout speed, full frame single read & 3500 frames s$^{-1}$ \\
Total noise for 1 ms of integration, looking at a & $0.4 \mathrm{e} \mathrm{pixel}^{-1}$ \\
$300 \mathrm{~K}$ scene & \\
Quantum efficiency & $>70 \%$ \\
Cold filters transmission (at $1.55 \mu \mathrm{m})$ & $81 \%$ \\
$H$-band filter cutoff & $1.739 \mu \mathrm{m}$ \\
Excess Noise Factor (ENF) & 1.25 \\
System gain & $0.59 \mathrm{ADU} / \mathrm{e}$ \\
Operating temperature & $80 \mathrm{~K}$ \\
\hline
\end{tabular}

at $1.55 \mu \mathrm{m}$ by the mean of a NIST-traceable calibrated diode. The result is $48 \pm 3 \%$. This value is somewhat in agreement with the theoretical specifications of $55 \%$ at this wavelength. Using the theoretical transmission and quantum efficiency values gives an incident background on the detector of $F_{\mathrm{BKG}_{\text {thermal }}}=$ 0.042 e frame $^{-1}$ pixel $^{-1}$.

\subsection{Warm optics setup}

The data set used for the model presented in this paper have been taken with the optical configuration used for on-sky observations, but with the instrument fed by an internal thermal white-light source (Fig. 1-right). In summary, the light is injected into a fiber whose output acts as the entrance slit of a simple spectrograph. A prism disperses the light over 12 pixels across the $H$-band. The image on the detector therefore consists of $3 \times 12$ illuminated pixels. The neighboring pixels are not illuminated by the flux coming from the fiber. In the following, we used only the value of a single pixel of the spectrum, which receives the flux from a sharp spectral band of approximately $0.05 \mu \mathrm{m}$ centered on $1.55 \mu \mathrm{m}$.

\section{Detector characterization}

\subsection{Mean-variance analysis}

The mean-variance method is a very common way of measuring the gain and the readout noise of a detector (Bohndiek et al.
2008). It is based on the fact that the temporal variance of a photometrically stable signal is proportional to the flux signal. The proportionality coefficient is the total gain multiplied by the excess noise factor. The interception of the fit with the y-axis gives the readout noise. To measure the system gain of our detector, we perform a mean-variance curve at an avalanche gain of 1 (i.e., without avalanche process).

We measure (Fig. 2) a system gain $G_{\text {sys }}=0.49 \mathrm{ADU} / \mathrm{e}$. We note that this is somewhat different from the expected system gain of $0.59 \mathrm{ADU} / \mathrm{e}$ provided on the data sheet from FLI. We dig into the calibration data of the manufacturer. Depending on the portion of the curve that we fit, the gain can vary from 0.42 to 0.61 . These differences may be explained by the nonlinearity of the detector at different flux levels. We do not study the effects of the nonlinearity in this paper because we focus on the low-lightlevel regime.

The mean-variance analysis also allows for the detector readout noise to be estimated as the floor noise obtained without illumination. The readout noise is $\approx 17$ ADU RMS, derived from the variance of $293 \mathrm{ADU}^{2}$ from the fit. Using the system gain, this corresponds to $\approx 35 \mathrm{e}$, which is comparable to the $45 \mathrm{e}$ measured with no APD gain by Finger et al. (2016) on a similar device and with a similar method.

We have identified that this detector noise is in fact partially correlated between the pixels. When using proper filtering (Lanthermann et al. 2018), we typically achieve detector noise as low as $\approx 12$ ADU RMS.

\subsection{Avalanche gain}

The avalanche gain is the mean value of electrons at the end of the avalanche process for one incident photo-electron. It represents the amplification of the incoming signal before the readout. To calibrate the avalanche gain, we measure the flux registered by the camera at different requested avalanche gains and divide it by the flux measured with no avalanche gain for the same incident flux. At the wavelength of $1.55 \mu \mathrm{m}$, the quantum efficiency of the camera is constant with the avalanche gain as shown in Fig. 6 of Finger et al. (2016). We double checked the temporal stability of the flux using a calibrated diode. Over the course of the experiment, the calibrated photometry of our light source was stable within a few percent.

Figure 3 displays the measured avalanche gain compared to the manufacturer calibration. The unity gain is used as a reference to compute the avalanche gain and therefore lies on the red 


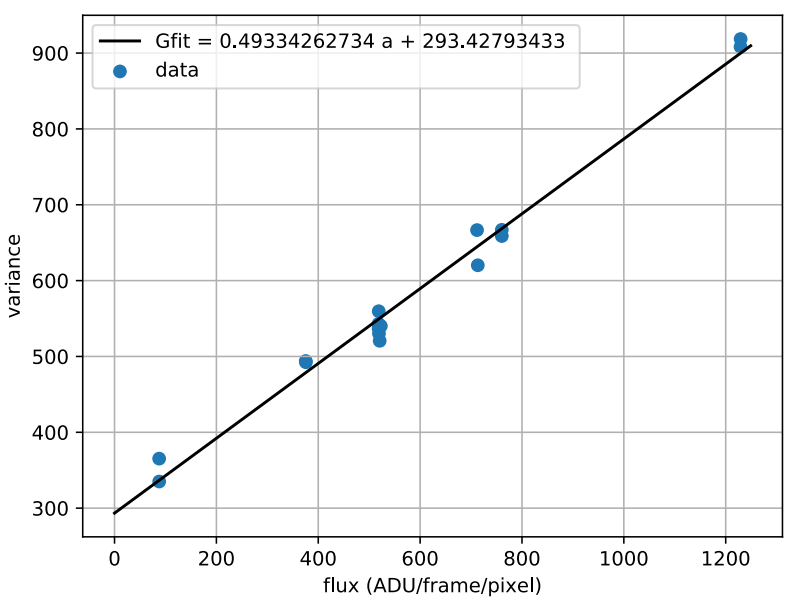

Fig. 2. Mean-variance curve at avalanche gain $=1$ (no avalanche process). The horizontal axis shows the mean flux in ADU/frame/pixel while the vertical axis gives the temporal variance. The blue dots are the data points. The black line is the fit of a linear function on the data points.

curve by definition. Our measured avalanche gain is consistently 0.93 times the avalanche gain calibrated by the manufacturer Although not presented in Fig. 3, we have verified that this relation applies for avalanche gain up to 150 .

The calibration from the manufacturer is based on a different method; it is a mean-variance analysis for each amplification gain. Our independent measurement confirms this calibration. The remaining discrepancy of few percent on the absolute gain is not an issue in our operational scenario. Neither the real-time fringe-tracking algorithms nor the instrument data-reduction algorithms rely on our absolute knowledge of the gain.

\subsection{Attempt of photon counting}

For a total gain of $50 \mathrm{ADU} / \mathrm{e}$ or higher, the signal of incoming individual photons should overwhelm the readout noise of $12 \mathrm{ADU}$ and therefore allows photon counting. This is especially true since the expected excess noise factor (ENF) is small (1.25). The ENF is the noise added by the avalanche process of the e-APD. The lower it is, the lower the add noise of the avalanche process. It has a limit of one for a noiseless avalanche process. The effect of the ENF on the photon counting was to spread the signal distribution of one photon. To explore this possibility of individual photon counting, we compute the histograms of the temporal sequences of the values of a single illuminated pixel. Typical histograms are shown in Fig. 4. We interpret the histogram of the background as the sum of (1) a Gaussian distribution of RMS 12 ADU corresponding to the detector readout noise; and (2) a small tail toward positive values corresponding to the few frames with a background photon (approximately 1 over 23 frames). The histogram measured in the low-flux regime shows a break at 25 ADU. We interpret this break as the transition between the zero-photon events and the one or more photon events. This means that we can derive the proportion of zerophoton events, hence the incoming flux. In the high-flux regime, the fraction of zero-photon events becomes negligible. The clear transition between the zero-photon events and events with one or more photons is no longer noticeable. Therefore, it appears that performing discretized photon-counting is not possible with this camera. In the following, we develop a simple model that reproduces these histograms and allows us to measure the excess noise factor.

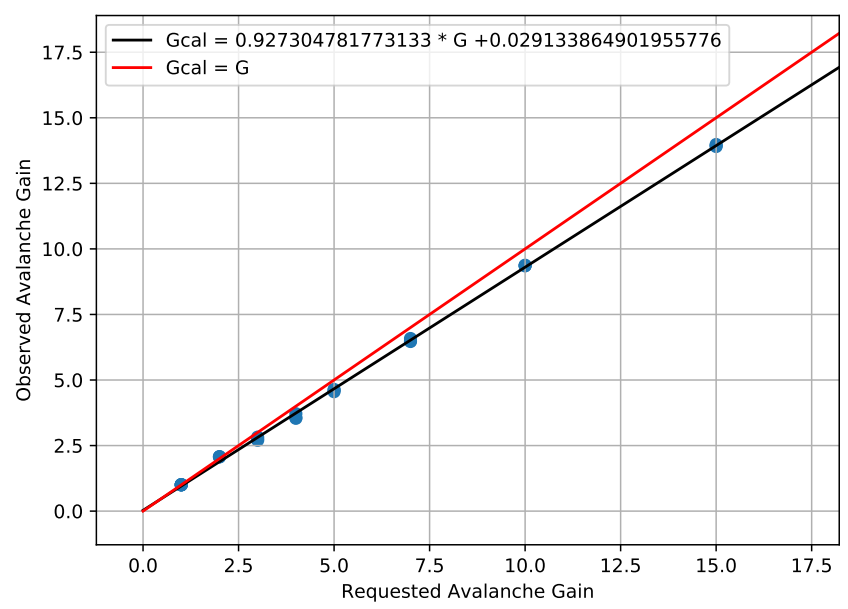

Fig. 3. Derived avalanche gain as a function of the avalanche gain provided by the manufacturer. The red line is the 1:1 relation. The black line is a linear fit, excluding the unity gain.

\section{Modeling the photonic signal}

We record histograms for various illuminations and various gains. Because of the very low flux levels we are interested in, it was unfortunately impossible to build an absolute calibration of the illumination.

\subsection{Model}

In the following, we use $\mathrm{F}$ to refer to the mean flux reaching the considered pixel and coming from the internal source, in e/frame/pixel. This is an unknown parameter. $F=0$ is by definition the mean of the histogram of the background counts (in green in Fig. 4). We developed a simple model with the goal being to reproduce the observed distributions $\mathrm{H}(\mathrm{ADU})$ as a function of the parameters $\mathrm{G}$ (gain in ADU/photon), the F (flux in photon/frame/pixel), and the ENF (excess noise factor). Our model is described by the following equation.

$$
H=\overbrace{B K G(\mathrm{ADU}, G)}^{(3)} * \overbrace{\sum_{\mathrm{p}}[P(p, F)}^{(2)} \cdot \overbrace{\left.M C\left(\mathrm{ENF}, \frac{\mathrm{ADU}}{G}\right)\right]}^{(1)},
$$

where the asterisk represents convolution, $B K G(\mathrm{ADU}, G)$ is the measured distribution without illumination, $P(p, F)$ is the Poisson probability of a $p$-photon event given a mean flux Flux, and

$$
M C=\overbrace{M(\mathrm{ENF}, \mathrm{ADU} / G) * M(\mathrm{ENF}, \mathrm{ADU} / G) * \ldots}^{p-1 \text { convolutions }}
$$

is the iterative self-convolution of the gain distribution because each avalanche generates its own avalanche gain distribution. This model is based on the following realistic assumptions and follows these steps:

1. The amplification gains of the individual photons summed up in a frame with $p$-photon are not correlated. Therefore the distribution of the $p$-photon frames is represented by $p-1$ convolutions of the gain distribution $M$.

2 . These $p$-photon frames are uncorrelated, therefore the combined distribution is a weighted sum according to the Poisson distribution for the desired flux.

3. The background flux is additive and uncorrelated with the illumination flux. Therefore the distribution given by the previous steps is convoluted by the distribution measured on the background. 




Fig. 4. Histograms of counts measured on a single pixel, with a lowflux illumination $\left(<1 \mathrm{e} \mathrm{frame}^{-1}\right.$ pixel $^{-1}$, darker blue $)$ and with a high flux illumination $\left(<3\right.$ e frame $\mathrm{fixel}^{-1}$, lighter blue). The total gain is $50 \mathrm{ADU} / \mathrm{e}$. The histogram without illumination is shown in green and represents the intrinsic statistic of the zero-photon events.

The key physical ingredient of this model is the gain distribution $M$.

\subsection{Prescription for the gain distribution}

The mean gain $\mathrm{G}$ and the ENF are intrinsic properties of $M$. A Gaussian distribution (Kardynał et al. 2008) truncated over $\mathbb{R}^{+}$ can only model low ENF values $(<1.2)$. This is because the fraction of nonphysical "negative amplifications" becomes numerically significant at larger ENF values. A decreasing exponential distribution truncated over $\mathbb{R}^{+}$(Tsujino et al. 2009) has a fixed ENF of 2.0. It is unsuited to reproduce the expected ENF of 1.25 of our camera.

The Gamma distribution is classically used to model the photon distribution in EMCCD cameras (Hirsch et al. 2013). It describes the process of multiplication of a single electron inside the pixel. The Gamma distribution is an intrinsically asymmetric function defined and normalized over $\mathbb{R}^{+}$. It is defined by two positive parameters, $k$ and $\theta$ (see Appendix A). The former parameter describes the shape of the function, while the latter describes its scale. These two parameters can be directly linked to the ENF and the mean gain by

$k=\frac{1}{\mathrm{ENF}-1}$,

and

$$
\theta=G(\mathrm{ENF}-1)
$$

The Gamma distribution allows spanning the entire range of ENF values between 1 and $+\infty$. Figure A.1 displays four examples of the Gamma distribution. Interestingly, the exponential distribution and the Gaussian distribution are special cases of the Gamma distribution. Therefore these two situations are included in our parameter space.

\subsection{Fit strategy}

Our model uses three free parameters $(G, E N F$, and the mean photonic flux $\mathrm{F}$ ) in order to reproduce the observed distribution $\mathrm{H}(\mathrm{ADU})$. The background distribution $B K G(\mathrm{ADU})$ is measured by turning off the internal source.
We first run a brute-force minimization by computing the reduced chi-squared $\chi_{\mathrm{r}}^{2}$ between the data and the model for each point in the cube $(G, \mathrm{ENF}, F)$. At the end of the brute force method, we run a classical gradient descent toward the best-fit parameters with the minimum $\chi_{\mathrm{r}}^{2}$ position in the cube as the starting point. The whole process on the 25 data files takes $\sim 3 \mathrm{~h}$ with 25 points on the grid for each parameter. We checked that this sampling is enough to sample the degeneracy by processing some data sets with 50 and 100 points on the grid for each parameter.

\section{Results and discussion}

\subsection{Best-fit quality}

Figure 5 shows the results of the minimization for a data set with a low flux level. The overall shape of the data is convincingly reproduced by the best-fit solution. The solution has no degeneracy and there is only one minimum for each parameter. This is because the fraction of zero-photon events is well constrained by the "bump" at zero. Knowing the flux, the mean on the histogram constrains the gain. Finally, the ENF is constrained by the shape of the histogram tail.

Appendix $\mathrm{C}$ shows all the results. The best-fit models convincingly reproduce the data for all flux and gain. This confirms that the Gamma distribution is an adequate model of the amplification process. As expected, for $F>3 \mathrm{e} \mathrm{frame}^{-1}$ pixel $^{-1}$, the degeneracy of the model increased significantly, as shown in Figs. C.3-C.5. This is due to the fact that the proportion of zerophoton events is no longer significant enough to constrain the flux with the data histograms. The $\chi_{\mathrm{r}}^{2}$ maps show that we have only a lower limit to F. As a consequence, we only have a higher limit for $\mathrm{G}$, and ENF is poorly constrained.

\subsection{Gain}

Figure 6 displays the best-fit gain versus the expected gain as calibrated by the manufacturer. The results obtained with different flux levels and gains are self-consistent: our best-fit gain $(\mathrm{G})$ is consistent with 0.71 times the expected gain from the manufacturer calibration $\left(G_{\text {man }}\right)$. We notice that the higher the flux level, the larger the uncertainties. This is due to the degeneracy at high flux levels. Interestingly, the best-fit gains for those highflux data are still consistent with the ones at low flux.

We analyzed several pixels in order to check the uniformity of the results in the matrix (see Appendix B). The results are consistent over the pixels and show a mean gain $G=0.70 \pm$ $0.04 \times G_{\operatorname{man}}$. This is in overall agreement with the fully independent calibrations presented in Sect. 3. With these more classical methods, we obtained a ratio of $G_{\text {sys measured }} / G_{\text {sys man }} \times G_{\text {ava ratio }}=$ $0.49 / 0.59 \times 0.93=0.77$ for the total gain, compared to the manufactured calibration. This also confirms that our simple model adequately captures the statistics of the amplification process.

\subsection{Excess noise factor}

Figure 7 shows the measured ENF as a function of the manufacturer gain. The median value of all the measured ENF is $1.49 \pm 0.12$. As expected, the uncertainty increases for measurements at higher flux but the error bars all remain compatible with the median value. As for the gain, we performed the same study on several pixels. The results are summarized in Appendix B. The mean ENF over the pixels is $1.47 \pm 0.03$.

The discrepancy between our measured ENF and the value reported in the literature is striking. Finger et al. (2016) measure 

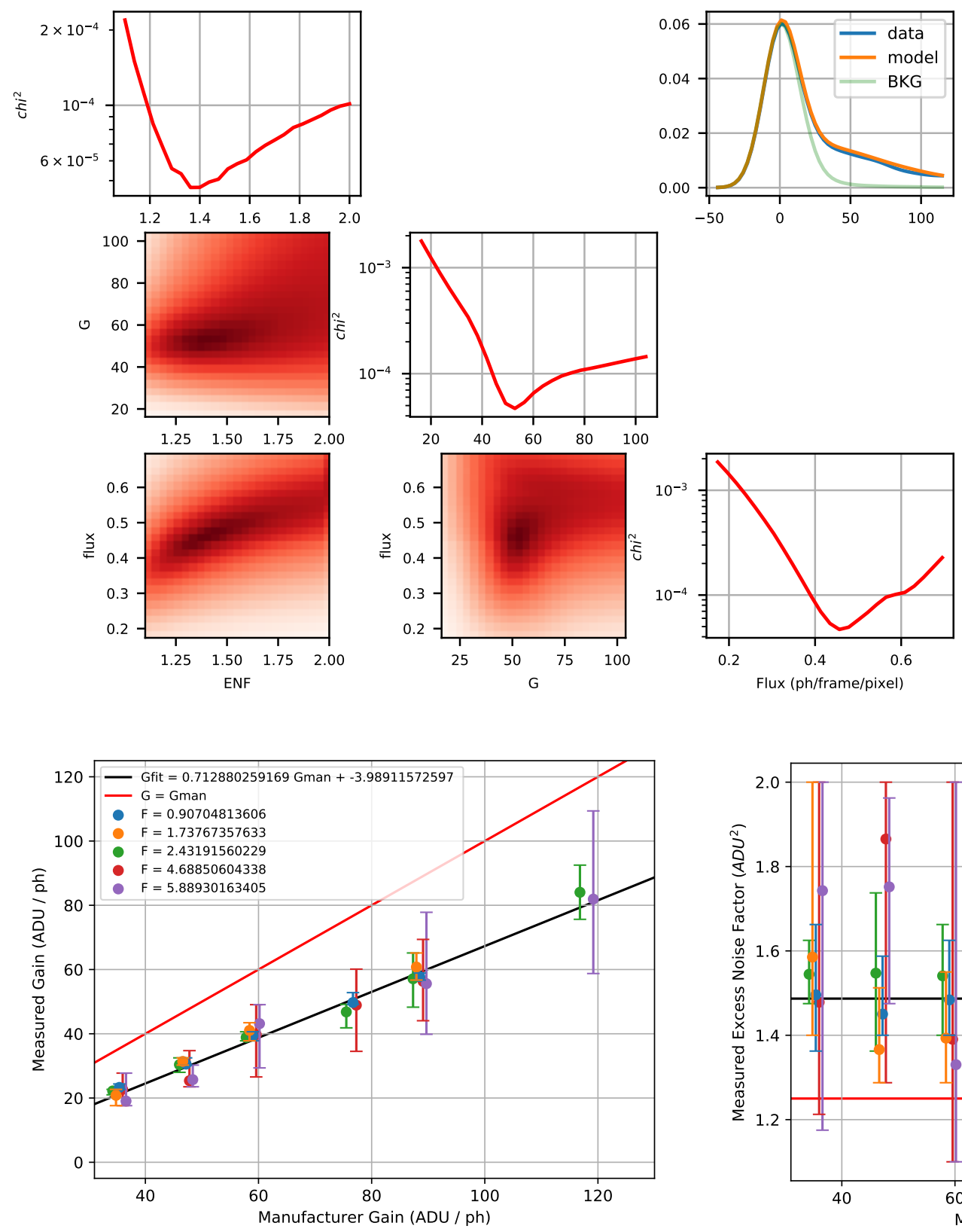

Fig. 6. Best-fit gain as a function of the expected gain from the manufacturer calibration. The black line is a linear fit, while the red line is the expected 1:1 relation. The different colors of the data points show the different flux estimated by the model-fitting.

an ENF of 1.3 at a temperature of $90 \mathrm{~K}$ and 1.1 at $40 \mathrm{~K}$. Their method to measure the ENF is independent of a gain distribution model. They control the illumination by using a black-body source located in front of the detector. They compare the noise on the detector with the photon noise expected for the controlled flux. Based on this calibration, the value expected by the manufacturer for our camera is 1.25 for an operating temperature of $80 \mathrm{~K}$.

The detailed study of a MARK13 SAPHIRA presented by Atkinson et al. (2018) gives interesting clues on the ENF. Their Fig. 6 shows a histogram of the detector signal with very low illumination (about 0.1 photon frame ${ }^{-1}$ pixel $^{-1}$ ). The histogram has a peak at the position of the zero-photon events whose shape is similar to the histogram without illumination, and a tail for the events with one or more photons. As in our measurements, data. different.

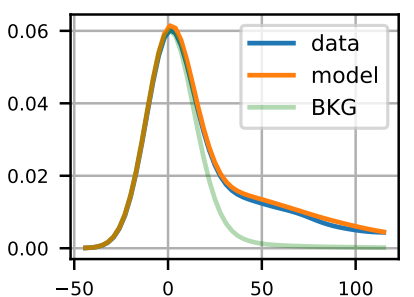

Fig. 5. Example of $\chi_{\mathrm{r}}^{2}$ minimization result. This example is from data with $\mathrm{Gman}=76.7$, the expected gain from the manufacturer calibration. The parameters that minimize the $\chi_{\mathrm{r}}^{2}$ are $F=0.46, G=$ 52.2, and $\mathrm{ENF}=1.38$. Diagonal: minimum $\chi_{\mathrm{r}}^{2}$ as a function of ENF (top left), $\mathrm{G}$ (middle), and $\mathrm{F}$ (bottom right), the two other parameters being free. Lower left: maps of minimum $\chi_{\mathrm{r}}^{2}$. Upper right: histogram for the data with illumination in blue, histogram of the background in light green, and histogram obtained with the parameters that minimize the $\chi_{\mathrm{r}}^{2}$ in orange.



Fig. 7. Best-fit ENF as a function of the manufacturer gain. Colors represent the flux estimated by the best-fit. The black line is the median value of measurements. The red line is the value from the manufacturer.

there is no separation between those two distributions. We run our model on the data extracted from Fig. 6 of Atkinson et al. (2018). The results are presented in Fig. C.6. The model reproduces adequately the shape of the observed histogram. The best fit $\mathrm{ENF}=1.45$ is consistent with the results obtained on our own

The first possibility is the somewhat arbitrary choice of the gain distribution model $M$ in our study. We also tried a Gaussian model defined over $\mathbb{R}^{+}$. The resulting ENF were not significantly

Another possibility is that the illumination contains photons at higher wavelengths. These photons are absorbed deeper in the pixel, possibly inside the multiplication region. This would create a less amplified signal, spreading the gain distribution. But our optical setup disperses the spectrum of the white-light source. Based on the spectral calibration of the instrument, we 
are sure that the pixels considered for this study only receive photons between 1.55 and $1.6 \mu \mathrm{m}$ coming from the white-light source. We remind the reader that the photons of the warm background are already taken into account in the background histogram.

Another hypothesis is that the signal of individual photon is temporally smeared. In Finger et al. (2016), the detector response time is of the order of $1 \mathrm{~ms}$. As the measurement of the ENF in the same paper is made at $1 \mathrm{kHz}$, the signal would not be smeared. In our experiment, we took data at $1900 \mathrm{~Hz}$, which is faster than the detector response time. But the claim is that the detector response time is dominated by the electron migration in the substrate and not by the avalanche process (Finger et al. 2016). Hence, it would delay the signal, not smear it. Another smearing occurs due to our specific readout mode, for photons arriving between consecutive readout that are averaged together into a single frame. We read an entire row several times before reading the others, and average these measurements. As we read 20 rows, it means that only $5 \%$ of the photons are smeared this way. This is too low to explain the difference between our value and the ones in the literature. Moreover, such an effect skews the distribution of gain toward the lower values (negative skewness), not toward the higher values (positive skewness) as seen in Fig. 6 of Atkinson et al. (2018) and across this paper.

The last possibility is that the results existing in the literature have been obtained for relatively high flux (several tens of photons per frame and per pixel), while our study and that of Atkinson focus on very low flux levels (less than 5 photons per frame and per pixel, and more typically less than 1 photon per frame and per pixel). We suspect that the physics of the detector is not the same when there is one or several electrons migrating in the amplification zone.

\subsection{Consequences for photon-counting}

As shown in Sect. 3.3, photon counting by fully separating the distributions of zero, single, or multiple photon events is not possible with our camera. This is due to the unexpected ENF of 1.47. However, Fig. 4 shows that we do see two different distributions at low flux: the one of the zero-photon events, and the one of the events with one or more photon. Therefore, one could define a threshold that would separate the frames with and without photons, with some statistical uncertainties. Computing this optimal threshold for each gain and each frame rate is out of the scope of this paper.

Although disentangling the zero-photon events from those with one or more photons may appear attractive, we did not implement this in the data-reduction pipeline of the instrument. Indeed, when dealing with a significant fraction of events with more than or equal to two photons, such a threshold dramatically complicates the un-biasing of the fringe bi-spectrum. A detailed discussion on the impact of the measurement statistics on the fringe bi-spectrum can be found in Basden \& Haniff (2004), Gordon \& Buscher (2012), and Garcia et al. (2016). We are currently upgrading the bi-spectrum de-biasing algorithm in the MIRCX pipeline based on our new understanding of the signal statistic presented in this study.

\subsection{Consequences on instrument performance}

We simulate the $\mathrm{S} / \mathrm{N}$ of the MIRC-X instrument for two realistic situations. First, we simulate a flux of $0.1 \mathrm{e} \mathrm{pixel}^{-1} \mathrm{~ms}^{-1}$ which is typically obtained on faint objects, such as young stellar objects (YSOs). These are the primary science goals of MIRC-X. The

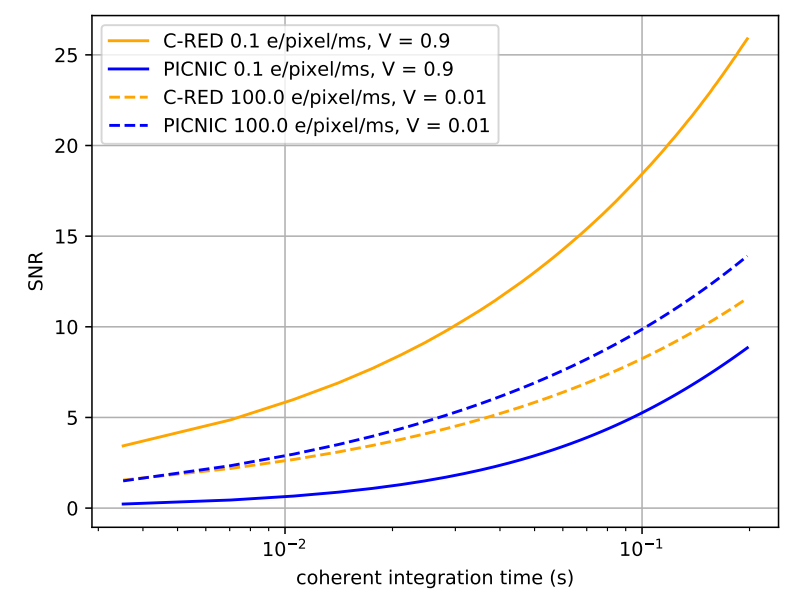

Fig. 8. Simulation of the $\mathrm{S} / \mathrm{N}$ as a function of the coherent integration time in two operational conditions of flux and fringe visibility, for the previous PICNIC camera of MIRC and the new C-RED ONE camera of MIRC-X.

fringe visibility is often high (close to 1) because the objects are unresolved. Secondly, we simulate a flux of $100 \mathrm{e} \mathrm{pixel}^{-1} \mathrm{~ms}^{-1}$ which is typical of bright objects, such as fully resolved stellar surfaces. These targets have very low visibility (e.g., 0.01). We estimate the detector and background noise by using actual measurements in background exposures obtained with the former PICNIC camera and with the C-RED ONE at an avalanche gain of 60 (best compromise between readout noise and tunneling noise; Lanthermann et al. 2018). We used the measured $\mathrm{ENF}=1.47$ for the C-RED ONE camera, and ENF $=1$ for the PICNIC camera that does not have additional noise from an avalanche process. For both cameras, the fringe signal is encoded into 100 pixels. Results are shown in Fig. 8.

The operational limit for fringe detection is defined by $S / N>3$. In the low-flux regime, the simulation shows an improvement of one decade in terms of necessary integration time between the former PICNIC camera and the new C-RED ONE. This corresponds to a gain in sensitivity of $2.5 \mathrm{mag}$. In the high-flux regime, the PICNIC camera is more efficient but the difference is relatively small in terms of limiting magnitude. For the specific case of averaging many exposures on bright targets with the goal being to reach a high S/N, the PICNIC camera remains theoretically advantageous because it does not suffer from the ENF. However, this is not true in practice because other effects quickly become dominant such as the piston noise or the calibration accuracy.

This simulation compares realistically with the results obtained on sky so far. The typical limiting magnitude of the instrument with the former PICNIC camera was $H \approx 5.5$. Since the upgrade, it has been possible to observe several YSOs and massive stars at magnitudes of $H \approx 7.5$. Overall, in terms of scientific productivity for MIRC-X, the sensitivity boost obtained in the low-flux regime largely overcomes the possible sensitivity losses in the high-flux regime.

\section{Conclusions}

We have characterized the first NIR C-RED ONE camera manufactured by the First Light Imaging company. This SAPHIRA electron avalanche photo-diode camera is now part of the MIRC-X instrument operating in the $H$ band and installed at the combined focus of the CHARA array. 
We have presented the characterization of this C-RED ONE camera in the operational scenario of the MIRC-X instrument (multiple readouts, $\mathrm{kHz}$ frame rate and low flux illumination). We first performed a classical analysis based on the meanvariance curve and mean flux level in order to determine the system gain and amplification gain. We then presented a simple but innovative modeling of the signal distribution from the camera at low flux level enabling us to estimate both the total gain and the excess noise factor brought by the amplification process. The model adequately reproduces the observed distributions of photon events for a large range of flux and gains. We also applied our model to existing literature data to confirm our measurements.

We measured a system gain and total gain slightly lower than those provided by the manufacturer. This result is observed consistently when using the mean-variance method and our model based on the signal distribution. Our modeling also revealed that the ENF (1.47) is significantly higher than the values in the literature for this type of detector (1.25). The reason for such a discrepancy could not be determined. After reviewing various possibilities, we proposed that the amplification process could behave differently in the low-flux and high-flux regimes.

From Sect. 5.5, we show that the C-RED ONE in the MIRC-X instrument brings a theoretical improvement in sensitivity of 2.5 mag in the $H$ band for faint objects, when its performances are comparable to the former PICNIC camera for bright objects. More generally, with an equivalent readout noise of $<0.3$ electrons at kilohertz frame rates computed from the background histograms (Fig. 4), we confirm the revolutionary performances of the camera with respect to the PICNIC or HAWAII technologies for high-frame-rate applications. In current operation, the camera has improved the limiting magnitude of our MIRC-X instrument by up to two magnitudes, the exact value depending on the atmospheric coherence time and the setup of the spectrograph. However, the observed higher excess noise factor is consistent with the fact that we cannot separate the events with different numbers of photons in the observed signal distribution.

Acknowledgements. We would like to thanks Marc-Antoine Martinod, Johan Rothman, and Romain Laugier for their interest in this work and the fruitful discussions that lead to the choice of the Gamma distribution for the gain distribution. We thank also FLI for their collaboration in the characterization and the comprehension of the camera. We also thank the University of Michigan for welcoming us and making this collaboration work possible. We finally thank CHARA and all the people that allowed us to collect data and observe. This work has been partially supported by the LabEx FOCUS ANR-11-LABX-0013. The research leading to these results has received funding from the European
Unions Horizon 2020 research and innovation programme under Grant Agreement 730890 (OPTICON) and Grant Agreement No. 639889 (ERC Starting Grant "ImagePlanetFormDiscs"). This work has been supported by a grant from Labex OSUG@2020 (Investissements d'avenir ANR10 LABX56). This work was supported by the Programme National de Physique Stellaire (PNPS) of CNRS/INSU co-funded by CEA and CNES. This work was supported by the Action Spécifique Haute Résolution Angulaire (ASHRA) of CNRS/INSU co-funded by CNES.

\section{References}

Atkinson, D., Hall, D., Jacobson, S., \& Baker, I. M. 2018, AJ, 155, 220 Basden, A. G., \& Haniff, C. A. 2004, MNRAS, 347, 1187

Beckmann, U., Connot, C., Heininger, M., et al. 2014, in Optical and Infrared Interferometry IV, Proc. SPIE, 9146, 91461W

Bohndiek, S. E., Blue, A., Clark, A. T., et al. 2008, IEEE Sens. J., 8, 1734

Feautrier, P., Gach, J. L., Guieu, S.. et al. 2014, in Adaptive Optics Systems IV, Proc. SPIE, 9148, 914818

Finger, G., Baker, I., Dorn, R., et al. 2010, Proc. SPIE, 7742, 77421K

Finger, G., Baker, I., Alvarez, D., et al. 2012, Proc. SPIE, 8453, 84530T

Finger, G., Baker, I., Alvarez, D., et al. 2016, Proc. SPIE, 9909, 99090D

Garcia, E. V., Muterspaugh, M. W., van Belle, G., et al. 2016, PASP, 128, 055004

Gies, D. R., ten Brummelaar, T. A., Anderson, M. D., et al. 2018, SPIE Conf. Ser., 10701, 1070102

Gordon, J. A., \& Buscher, D. F. 2012, A\&A, 541, A46

GRAVITY Collaboration (Abuter, R., et al.) 2017, A\&A, 602, A94

Greffe, T., Feautrier, P., Gach, J. L., et al. 2016, Optical and Infrared Interferometry and Imaging V, 9907, 99072E

Guieu, S., Feautrier, P., Zins, G., et al. 2014, Optical and Infrared Interferometry IV, 9146, 91461N

Haguenauer, P., Abuter, R., Andolfato, L., et al. 2012, in Optical and Infrared Interferometry III, Proc. SPIE, 8445, 84450D

Hirsch, M., Wareham, R. J., Martin-Fernandez, M. L., Hobson, M. P., \& Rolfe, D. J. 2013, PLoS One, 8, 1

Kardynał, B. E., Yuan, Z. L., \& Shields, A. J. 2008, Nat. Photonics, 2, 425

Kraus, S., Monnier, J. D., Anugu, N., et al. 2018, Proc. SPIE, 10701, 10701

Lanthermann, C., Bouquin, J. B. L., Anugu, N., Monnier, J., \& Kraus, S. 2018, Proc. SPIE, 10709, 10709

Le Bouquin, J.-B., Berger, J.-P., Lazareff, B., et al. 2011, A\&A, 535, A67

Monnier, J. D., Pedretti, E., Thureau, N., et al. 2006a, in SPIE Conf. Ser., Proc. SPIE, 6268, 62681P

Monnier, J. D., Pedretti, E., Thureau, N., et al. 2006b, Proc. SPIE, 6268, 6268

Monnier, J. D., Pedretti, E., Thureau, N., et al. 2012, Proc. SPIE, 8445, 8445

Monnier, J. D., Bouquin, J.-B. L., Anugu, N., et al. 2018, Proc. SPIE, 10701, 10701

Pedretti, E., Millan-Gabet, R., Monnier, J. D., et al. 2004, PASP, 116, 377

Scott, N. J., Lhomé, E., ten Brummelaar, T. A., et al. 2014, in Optical and Infrared Interferometry IV, Proc. SPIE, 9146, 91461A

ten Brummelaar, T. A., Sturmann, J., McAlister, H. A., et al. 2012, in Optical and Infrared Interferometry III, Proc. SPIE, 8445, 84453C

ten Brummelaar, T. A., Gies, D. G., McAlister, H. A., et al. 2016, Proc. SPIE, 9907, 9907

Tsujino, K., Akiba, M., \& Sasaki, M. 2009, IEEE Electron Device Lett., 30, 24 


\section{Appendix A: Gamma distribution}
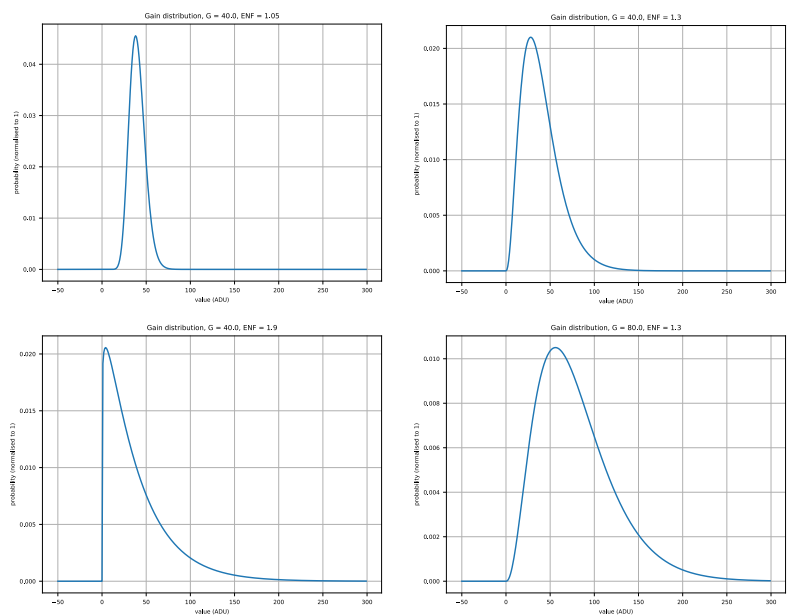

Fig. A.1. Different examples of gain distribution modeled with the Gamma distribution. Upper-left corner: $\mathrm{ENF}=1.05$ and $G=40$. Upper-right corner: $\mathrm{ENF}=1.3$ and $G=40$. Lower-left corner: $\mathrm{ENF}=1.9$ and $G=40$. Lower-right corner $: \mathrm{ENF}=1.3$ and $G=80$.

Figure A.1 shows four examples of the Gamma distribution for different values of avalanche gain and ENF. In the upper-left corner, we see a Gaussian distribution. In the lower-left corner, we are in the regime of an exponential distribution. The probability density function of the Gamma distribution is defined by

$f(x, k ; \theta)=\frac{x^{k-1} e^{-x / \theta}}{\theta^{k} \Gamma(k)}$

for $x>0$ and $k, \theta>0$, and where $\Gamma(k)$ is the gamma function. For this distribution, the $k$ parameter describes the shape of the distribution and $\theta$ parameter describes the scale of the distribution.

\section{Appendix B: Results from different pixels}

Table B.1. Results of gain and ENF for five different pixels.

\begin{tabular}{lll}
\hline \hline Pixel coordinate & $G / G_{\operatorname{man}}$ & $\mathrm{ENF}$ \\
\hline$(67 ; 7)$ & 0.72 & 1.49 \\
$(67 ; 9)$ & 0.73 & 1.49 \\
$(66 ; 9)$ & 0.71 & 1.49 \\
$(67 ; 11)$ & 0.69 & 1.44 \\
$(66 ; 13)$ & 0.63 & 1.43 \\
\hline
\end{tabular}

Table B.1 summarizes the results obtained for five different pixels, in order to quickly assess the homogeneity of the result across the detector.

The coordinates are measured within the $320 \times 20$ pixel window. The gain factor is computed with the linear fit of the gain of the best-fit model as a function of the manufacturer gain (Fig. 6). The ENF is obtained with the median of the different ENF measured by the model for various fluxes (Fig. 7). The plots shown in the main sections of the paper were for pixel $(66 ; 9)$. Table B.1 shows that we obtain similar values for other pixels.

\section{Appendix C: Different minimization results}

This appendix presents the results of minimizations for different typical configurations. Figure C.1 is for a low-flux and low-gain configuration. Figure C.2 is for a low-flux and high-gain configuration. Figure C. 3 is for a high-flux and low-gain configuration. Figure C. 4 is for a high-flux and high-gain configuration. Figure C.5 is for a high flux at the maximum gain used for this study. Figure C.6 is for the data from Fig. 6 of Atkinson et al. (2018). 
Brute force chi2 map, Gman $=47$, RMS background $=13.03$ minimum chi2 for $F=0.56, G=30.31$, ENF $=1.4$

$\mathrm{G} / \mathrm{RMS}=2.33$
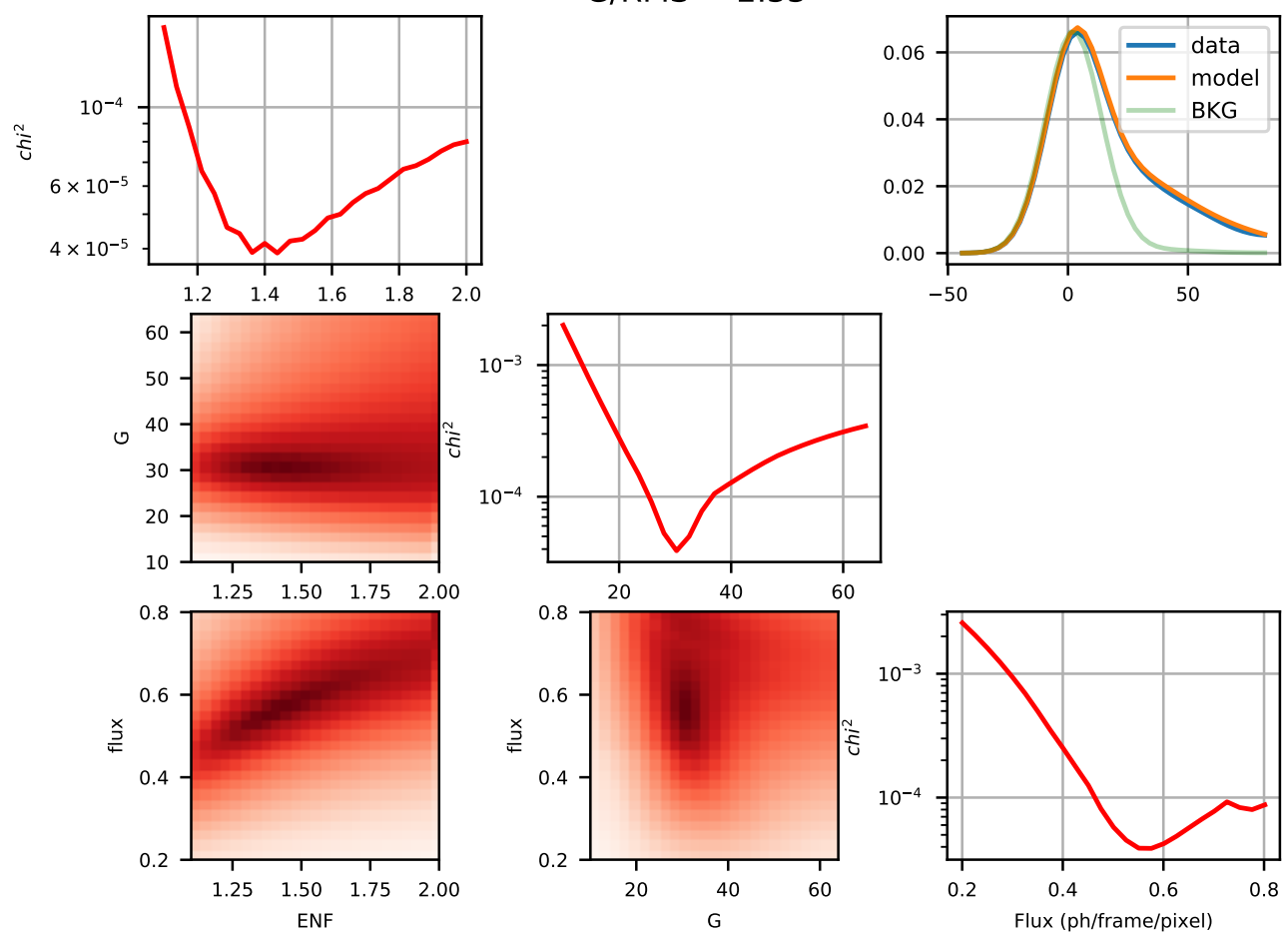

Fig. C.1. $\chi_{\mathrm{r}}^{2}$ minimization result example for low flux, low gain.

Brute force chi2 map, Gman $=88$, RMS background $=19.23$ minimum chi2 for $F=0.57, G=55.65, E N F=1.4$

$\mathrm{G} / \mathrm{RMS}=2.89$
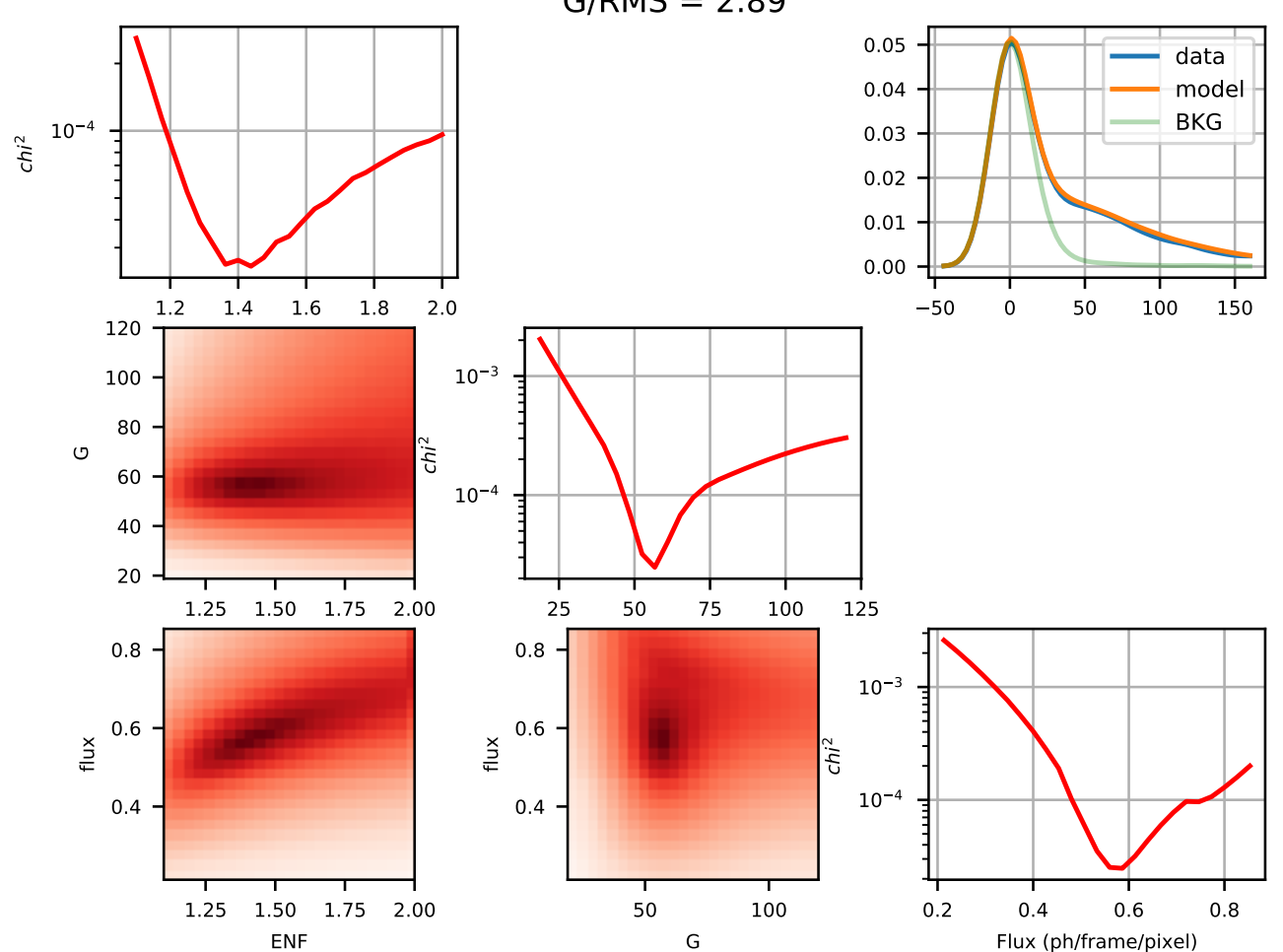

Fig. C.2. $\chi_{\mathrm{r}}^{2}$ minimization result example for low flux, high gain. 
C. Lanthermann et al.: Modeling the SAPHIRA/C-RED ONE camera at low flux level

Brute force chi2 map, Gman $=47$, RMS background $=13.77$ minimum chi2 for $\mathrm{F}=2.98, \mathrm{G}=31.76, \mathrm{ENF}=1.31$

$\mathrm{G} / \mathrm{RMS}=2.31$


Fig. C.3. $\chi_{\mathrm{r}}^{2}$ minimization result example for high flux, low gain.

Brute force chi2 map, Gman $=88$, RMS background $=19.7$ minimum chi2 for $F=3.01, G=57.81, E N F=1.45$

$\mathrm{G} / \mathrm{RMS}=2.93$
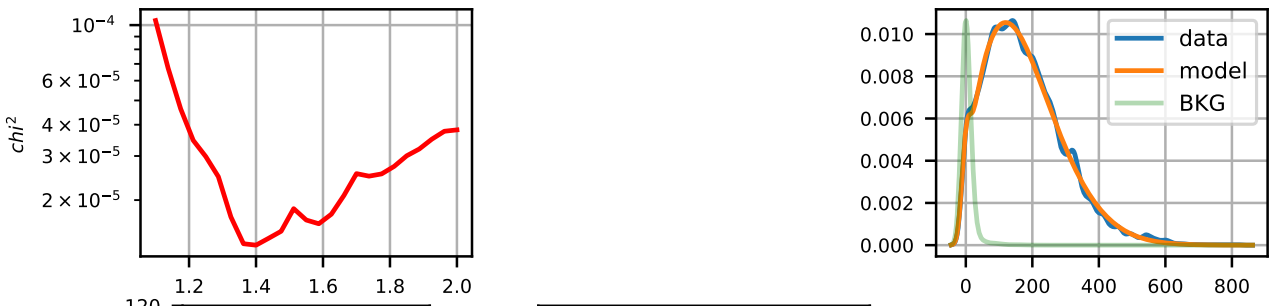

৩
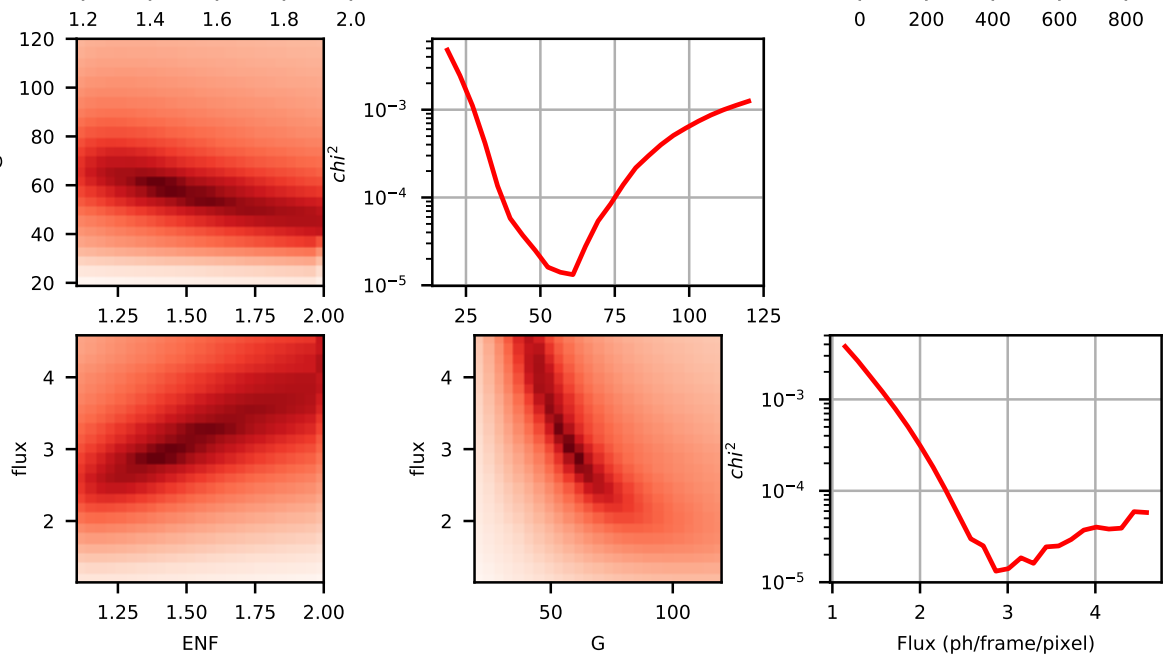

Fig. C.4. $\chi_{\mathrm{r}}^{2}$ minimization result example for high flux, high gain. 
Brute force chi2 map, Gman $=118$, RMS background $=25.77$ minimum chi2 for $F=3.18, G=69.52, E N F=1.53$

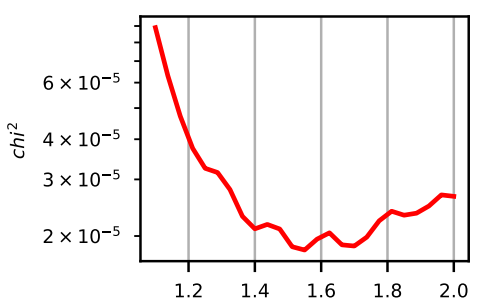

$\mathrm{G} / \mathrm{RMS}=2.7$
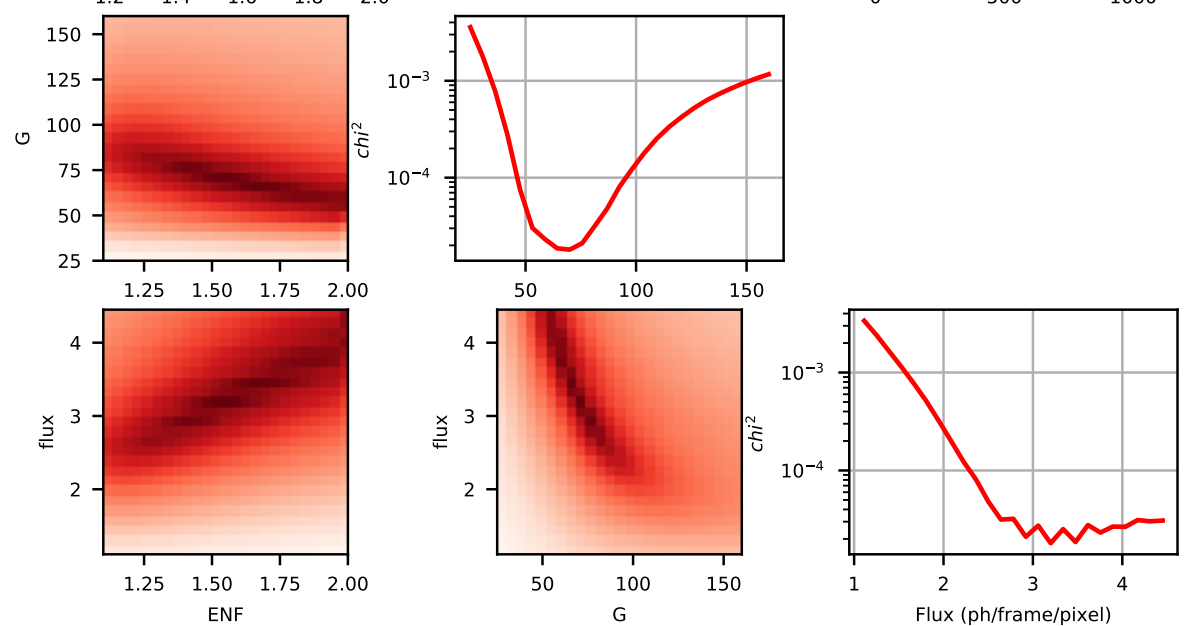

Fig. C.5. $\chi_{\mathrm{r}}^{2}$ minimization result example for high flux, highest gain.

Brute force chi2 map, RMS background $=8.97$ minimum chi2 for $F=0.07, G=58.66, E N F=1.45$


$\mathrm{G} / \mathrm{RMS}=6.54$
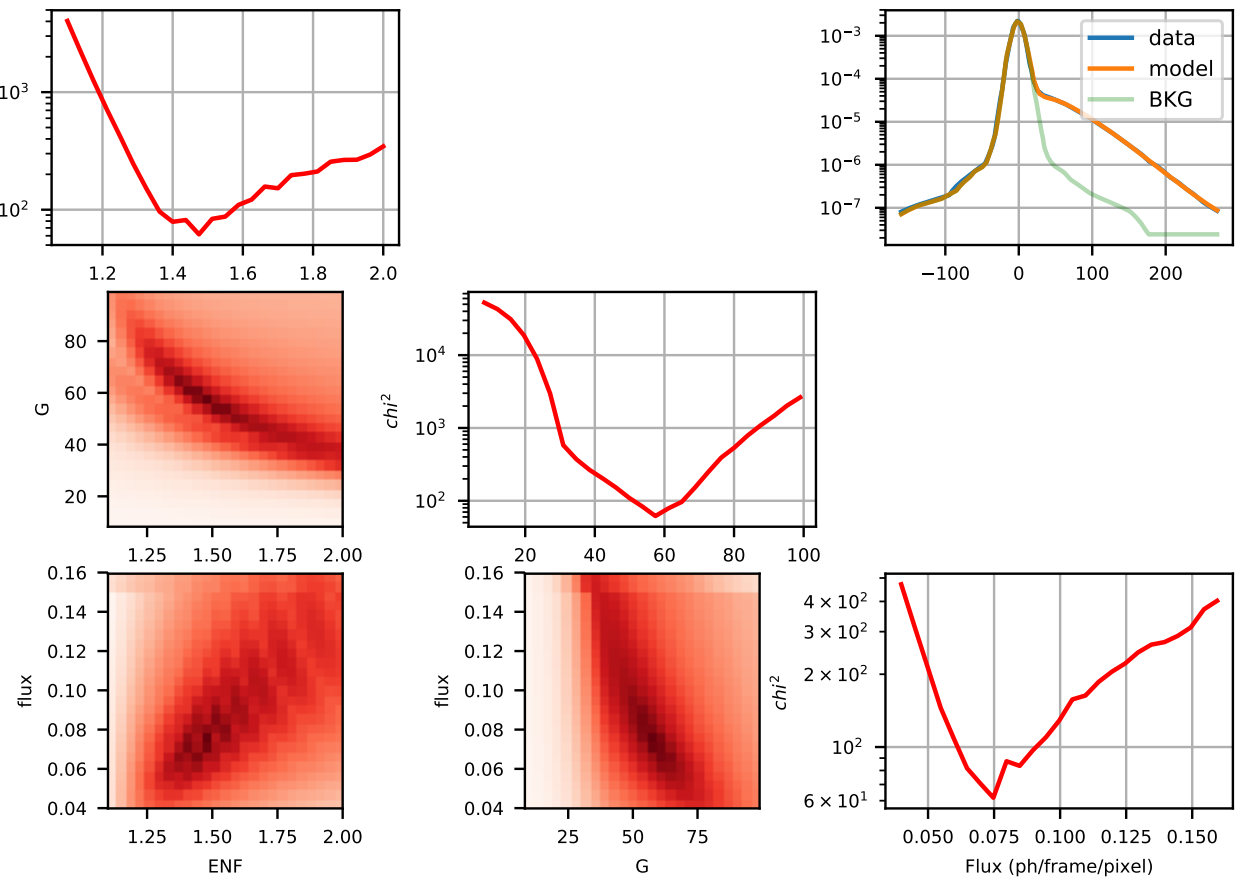

Fig. C.6. $\chi_{\mathrm{r}}^{2}$ minimization result for Atkinson et al. (2018) data. 